\title{
HTA: How to assess?
}

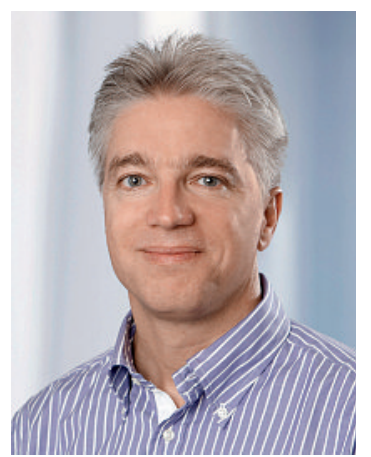

Zehn Prozent Leistungssteigerung während einer 6-minütigen Gehstrecke entsprechen gemäss Bundesgericht nicht dem Gebot der Wirksamkeit einer Behandlung. Wenn dann diese Leistungsverbesserung zu einem Preis von 500000 Franken pro Patient pro Jahr erzielt wird, ist zudem auch die Wirtschaftlichkeit nicht gegeben. Etwa so lässt sich das Bundesgerichtsurteil zum Fall eines Patienten mit Morbus Pompe, einer seltenen Muskelerkrankung, die mit dem Medikament Myozyme ${ }^{\circledR}$ behandelt werden kann, zusammenfassen. Zwar ist es nicht das erste Mal, dass die höchste richterliche Instanz der Schweiz Stellung nimmt zur Frage der Verhältnismässigkeit von Kosten und Nutzen einer medizinischen Massnahme. Zum ersten Mal aber beurteilt sie explizit die Übernahme einer medikamentösen Therapie eines Off-label-Medikaments (nicht auf der Spezialitätenliste).

In letzter Zeit mehren sich die juristischen Stellungnahmen zu Kosten-Nutzen-Fragen unter der Berücksichtigung von Artikel 32 des KVG (Wirksamkeit, Zweckmässigkeit, Wirtschaftlichkeit; auch als WZW bezeichnet). Konkret zeichnet sich immer mehr in der Rechtsprechung eine Obergrenze von 100000 Franken pro gerettetes Lebensjahr ab. Was heissen nun solche Urteile für das schweizerische Gesundheitswesen? Einiges. Grundsätzlich wirkt es auf den ersten Blick irritierend, dass sich Juristen über die Wirksamkeit einer medizinischen Behandlung äussern. Ist es nicht gerade dieser Aspekt der WZW-Kriterien, der bisher die unbestrittene Domäne der praktizierenden und forschenden Ärzteschaft darstellte? Wir sind ja letztlich diejenigen, die täglich mit den Patienten konfrontiert sind und die über die Informationen verfügen, die zur Beurteilung der Frage der Wirksamkeit notwendig sind.

\section{Bundesgerichtsentscheid zu Off-label-Medikament: Was darf ein gerettetes Lebensjahr kosten?}

Um sich zu Fragen der Wirksamkeit zu äussern, sind wir gefordert, die Behandlungsergebnisse zu dokumentieren und im Sinne der Beurteilung der Outcome-Qualität nach wissenschaftlich anerkannten Methoden zu analysieren. Das alleine genügt aber nicht, die so gemessene Wirksamkeit muss nun in den Kontext der verbleibenden ZW-Kriterien eingebettet werden. Wenn wir dann noch ethische und ge- sellschaftliche Aspekte miteinbeziehen sind wir bei einem Health Technology Assessment oder HTA.

Im Gegensatz zu manchen Nachbarländern ist in der Schweiz im Bereich HTA noch vieles unklar. Die grundsätzlichen Fragen, wer macht was, wie und mit welchen Konsequenzen, sind bisher ungeklärt. Der Bundesrat sah diesen Koordinationsbedarf und sprach sich letztes Jahr für eine Motion unseres Vizepräsidenten und Nationalrates Ignazio Cassis aus. Diese fordert die Schaffung von Rahmenbedingungen für ein unabhängiges nationales HTA-Institut. Bis es aber so weit ist, sind verschiedene Akteure bereits aktiv geworden. Unter dem klingenden Namen SwissHTA haben die Interpharma und santésuisse zu Arbeitsgruppensitzungen eingeladen, mit dem Ziel, Konsens zu schaffen. Die FMH wurde ebenfalls angefragt, sich zu beteiligen. Aufgrund des einseitigen Diktats, vor allem der Interpharma, sowie der fehlenden Einflussmöglichkeit, vor allem in der Auswahl der Themen, der Experten und der Arbeitsweise, hat sich die FMH entschieden, vorerst nicht mitzumachen.

Hingegen engagiert sich die FMH sinnvollerweise im Medical Board (www.medical-board.ch). Als einer der drei Träger, zusammen mit der GDK und der SAMW, können wir hier die Themen mitbestimmen und vor allem unsere Experten aus den Fachgesellschaften einbringen. Dieser Einbezug der Ärzteschaft verbessert die Qualität der Evaluationen deutlich und ist mehr als sinnvoll.

\section{Kosten und Nutzen von medizinischen Leistungen: Die FMH engagiert sich im Medical Board.}

So oder so bleibt viel zu tun, und nebst den brennenden Fragen um die Organisation und Koordination der HTA in der Schweiz dürfen wir die Patienten nicht vergessen, die das Pech haben, an einer seltenen Erkrankung zu leiden, und die nur mit teuren Off-label-Medikamenten behandelt werden können. Wer wird sich ihrer annehmen, wer legt die Obergrenze einer medizinischen Behandlung fest? Das sind Fragen, bei denen nicht nur die Akteure im Gesundheitswesen gefragt sind, sondern zu denen sich auch die Gesellschaft äussern muss, mit oder ohne HTA.

Dr. med. Daniel Herren MHA, Mitglied des Zentralvorstandes der FMH, Verantwortlicher für das Ressort DDQ

\section{Literatur}

Was darf ein Leben kosten? NZZ 23.12.2010.

BGE 9c_334/2010.

BGE 9c_56/2008. 